\title{
Integrated Analysis of microRNA-mRNA Network Predicts Potential Regulators of Atrial Fibrillation in Human
}

\author{
Rong Wang1,2, Emre Bektik ${ }^{2,3, \#, ~ P h r a e w ~ S a k o n ~}{ }^{4}$, Xiaowei Wang', Shanying Huang', Xiangbin Meng ${ }^{5}$, Mo Chen ${ }^{6}$, \\ Wenqiang Han ${ }^{1}$, Jie Chen ${ }^{7}$, Yanhong Wang ${ }^{8}$ and Jingquan Zhong1,,*
}

1 The Key Laboratory of Cardiovascular Remodeling and Function Research, Chinese Ministry of Education, Chinese National Health Commission and Chinese Academy of Medical Sciences, The State and Shandong Province Joint Key Laboratory of Translational Cardiovascular Medicine, Department of Cardiology, Qilu Hospital, Cheeloo College of Medicine, Shandong University, 107 Wenhuaxi Road, Jinan, Shandong 250012, China; wangrong@qiluhospital.com; wxw11130@163.com; huangshy2002@163.com; 742415985@qq.com;

2 Department of Cardiology, Boston Children's Hospital, Harvard Medical School, 300 Longwood Avenue, Boston, MA 02115, USA.

3 Department of Medicine, Cardiovascular Division, Brigham and Women's Hospital, Harvard Medical School, Boston, MA 02115, USA; ebektik@bwh.harvard.edu

4 Cancer and Blood Disorders Center, Boston Children's Hospital and Dana-Farber Cancer Institute, Harvard Medical School, Boston, MA 02215, USA; psakon@syr.edu

5 Department of Cardiac Surgery, Qilu Hospital, Cheeloo College of Medicine, Shandong University, 107 Wenhuaxi Road, Jinan, Shandong 250012, China; henry2008meng@hotmail.com

6 Department of Operating Room, Qilu Hospital, Cheeloo College of Medicine, Shandong University, 107 Wenhuaxi Road, Jinan, Shandong 250012, China; 394426697@qq.com

7 People's Hospital of Dingtao District, Heze City, Shandong Province, 056, Section A, Zhanqian Road, Dingtao District, Heze , Shandong, 274100, China; LPLCJL@126.COM

8 Department of Anesthesia and Surgery, Provincial Hospital of the First Medical University of Shandong Province, No. 324 Jingwu Weiqi Road, Jinan, Shandong, 250021, China; 18168889172@163.com

9 Department of Cardiology, Qilu Hospital (Qingdao), Cheeloo College of Medicine, Shandong University, 758 Hefei Road, Qingdao, Shandong, 266035, China; 18560086597@163.com

\# Correspondence: ebektik@bwh.harvard.edu (E.B.) or 18560086597@163.com (J.Z.)

\begin{abstract}
Atrial fibrillation (AF) is a form of sustained cardiac arrhythmia and microRNAs (miRs) play crucial roles in pathophysiology of AF. To identify novel miR-mRNA pairs, we performed RNAseq from atrial biopsies of AF and non-AF patients. Differentially expressed miRs (11-down and 9-up) and mRNAs (95-up and 82-down) were identified and hierarchically clustered in a heat-map. Subsequently, GO, KEGG, and GSEA analyses were run to identify deregulated pathways. Then, miR targets were predicted in miRDB database, and a regulatory network of negatively correlated miR-mRNA pairs was constructed using Cytoscape. To select potential candidate genes from GSEA analysis, top-50 enriched genes in GSEA were overlaid with predicted targets of differentially deregulated miRs. Besides, protein-protein-interaction (PPI) network of enriched genes in GSEA was constructed, and subsequently GO and canonical pathway analyses were run for genes in PPI network. Our analyses showed that TNF- $\alpha$, p53, EMT, and SYDECAN1 signaling were among the highly affected pathways in AF samples. SDC-1 (syndecan-1) was the top-enriched gene in p53, EMT, and SYDECAN1 signaling. Consistently, SDC-1 mRNA and protein levels were significantly higher in atrial samples of AF patients. Among negatively correlated $\mathrm{miRs}$, miR-302b-3p was experimentally validated to suppress SDC- 1 transcript levels. Overall, our results suggested that miR-302b-3p/SDC-1 axis may involve in pathogenesis of AF.
\end{abstract}

Keywords: heart disease; atrial fibrillation; atrial fibrosis; transcriptome; microRNA; RNA sequencing; syndecan-1; miR-302

\section{Introduction}

Atrial fibrillation (AF) is the most common form of cardiac arrhythmia in humans and often characterized by rapid and irregular beating of the atria [1,2]. AF affected 
$\sim 33.5$ million people only in 2010 worldwide and $\sim 5$ million new patients each year [3,4]. Prevalence of AF progressively increases in the aging society, leading to reduced life quality and/or elevated mortalities [5,6]. It was reported that averagely 130,000 AF patients die in the USA [7] and that AF affected 2-3\% of European population [4,8,9]. Also, AF is estimated to affect $\sim 72$ million people in Asia and patients with AF-related strokes to reach 2.9 million in the next 30 years $[3,10]$.

AF often starts by a trigger in a vulnerable substrate that leads to short episodes of rapid ectopic firing and re-entry of electrical signal in the atria. Over the course of time, AF may turn into a permanent condition through atrial remodeling process, which is central to AF [11]. Structural remodeling in atria is often identified by atrial fibrosis, dilation, and abnormal conduction of electrical pulses while electrical remodeling results with abnormal impulse generation due to ion channel dysfunction [12,13]. Current therapeutic approaches have limited efficacy and adverse effects in treatment of AF due to lack of molecular understanding of AF substrates and mechanism $[13,14]$. Therefore, there is an urgent need to expand mechanistic knowledge on AF development and progression.

Recent developments in AF research identified microRNAs (miRs) as novel regulators of AF $[15,16]$. MiRNAs are classified as a subtype of short non-coding RNAs with typical length of 18-24 nucleotides, and can negatively regulate expression of mRNA transcripts of target genes [17] with exception that some may directly regulate protein function, e.g. ion channels $[18,19]$. After initial discovery of miR-1 function in heart development, a large amount of research efforts focused on investigating miRNAs and their function in cardiovascular system. Indeed, a large number of miRNAs has been found to abnormally express in various cardiac disorders [20-22]. Gene expression of patient samples with AF history and other functional studies identified miRNAs in deregulation of ion channels, leading to electrical remodeling (15-17). Structural remodeling through cardiac fibrosis was also shown to be regulated by miRNAs (18-19). However, current knowledge of miRNA function in AF is limited and requires more research. Thus, analysis of miRNA and target gene expression networks can improve our knowledge on regulatory mechanisms of miRNAs in AF induction and progression $(20,21)$.

In this study, we aimed to predict novel miR-mRNA pairs that may play roles in pathogenesis of AF. Thus, we performed RNA sequencing of miRNAs and mRNAs from atrial samples of AF and non-AF patients (Figure 1). Several analyses including gene ontology (GO), Kyoto encyclopedia of genes and genomes (KEGG) pathway, and gene-set enrichment analyses (GSEA) were run for differentially expressed genes (DEGs) and top-enriched genes in GSEA of DEGs. Then, we profiled interaction-network of negatively correlated miRNA-mRNA pairs using Cytoscape. Our results revealed 15-gene and 13-miR candidates, among which gene expression levels of miR-302b-3p and SDC-1 were experimentally confirmed to be negatively correlated in atrial tissue samples. Besides, overexpression of miR-302b-3p by mimics significantly reduced SDC- 1 levels in vitro. SDC-1 is known for its function in cardiac fibrosis [23,24], and our analyses found SDC-1 in close interaction with AF-associated genes, e.g. SELE. Briefly, our results suggest that miR-302b-3p/SDC-1 axis may function in AF via modulating atrial fibrosis. 


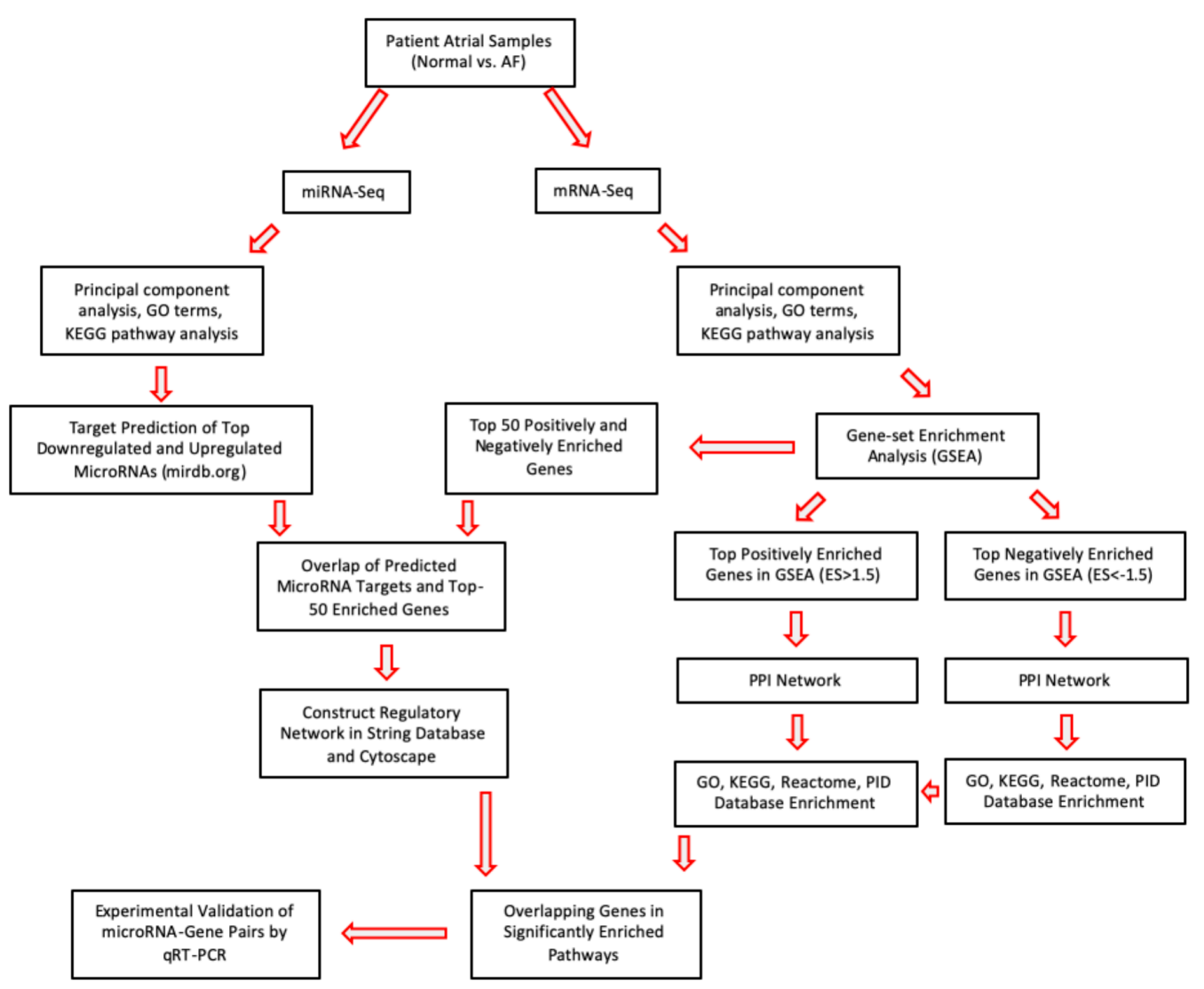

Figure 1. Flowchart of RNAseq Experimental and Target Prediction Strategy.

\section{Results}

\subsection{Analysis of Differentially Expressed miRNAs and mRNAs in AF}

Our miRNAseq of both control and AF groups detected total raw-read counts of $>42 \times 10^{6}$ miRs and clean-read counts of $>33 \times 10^{6}$ miRs with a cut-off sequence length of $\geq 18$ nucleotides. Also, more than $49 \times 10^{6}$ raw-read counts of mRNAs were detected in both control and AF groups. Principal component analysis of samples from AF or non-AF patients showed that each group was prominently clustered for miR-seq (Figure $2 \mathrm{~A}$ ) and mRNA-seq (Figure 3A). mRNA (Figure 2B) and miR (Figure 3B) expression distribution in each sample was shown in terms of $\log _{10}(\mathrm{fpkm})$. Total of 1,725 miRs and 17,087 genes were detected in our RNAseq. Differentially expressed genes (DEGs) or miRs with a threshold of $\log _{2}$ (Fold-change) $>1$ and a statistical significance of $p<0.05$ in both miR-seq (Figure 2C) and mRNA-seq (Figure 3C) were separately identified. Significantly differential expression levels of mRNAs (Figure 2D) or miRs (Figure 3D) were visualized in heat maps. There were 9 upregulated and 30 downregulated miRs (Figure 2A-D) and 95 upregulated and 82 downregulated mRNAs (Figure 3A-D), which were significantly differentially expressed. GO and KEGG pathway analysis found that various pathways were affected in AF (Figure 2E-F and Figure 3E-F). Among the top upregulated pathways, TGF- $\beta$ was one of the highly upregulated ones in miR-seq (Figure $2 \mathrm{E}$ ). On the other hand, mRNA-seq found cell adhesion molecules and TNF- $\alpha$ signaling pathway among the highly enriched pathways (Figure 3E). 
A

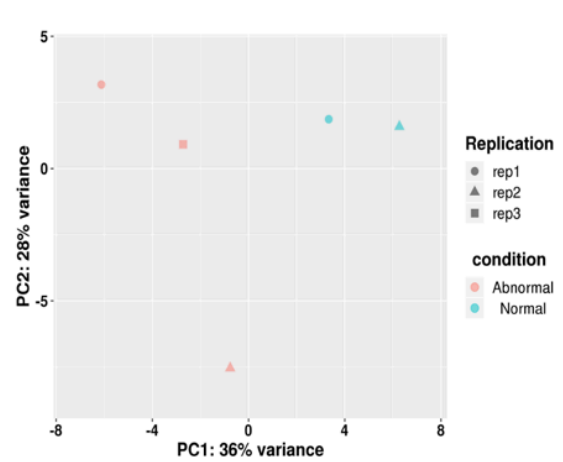

C
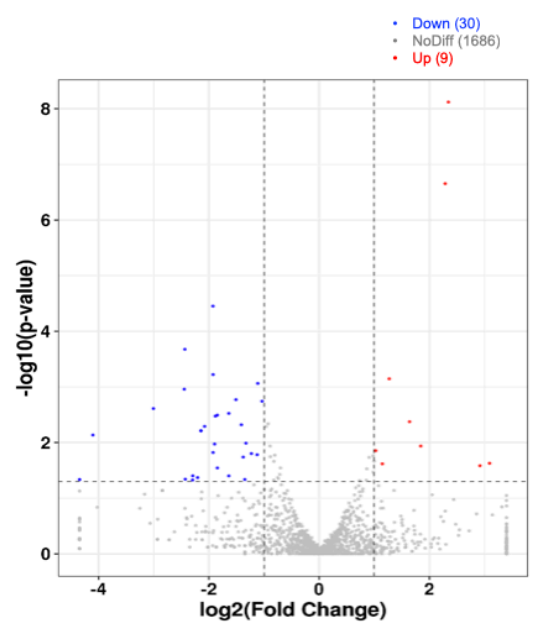

$\mathbf{E}$

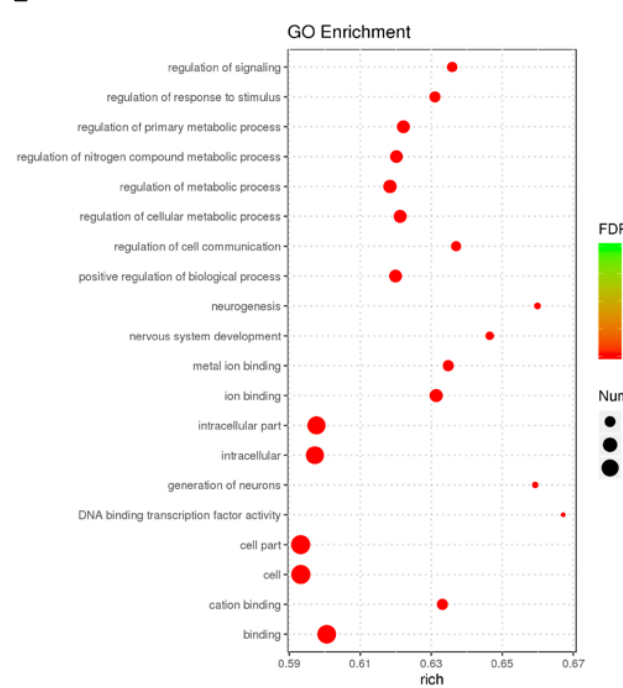

B

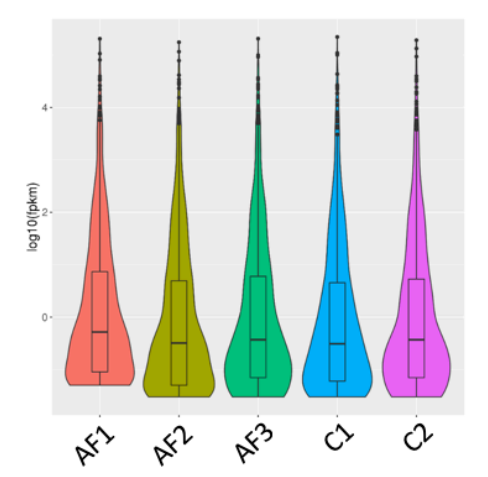

D

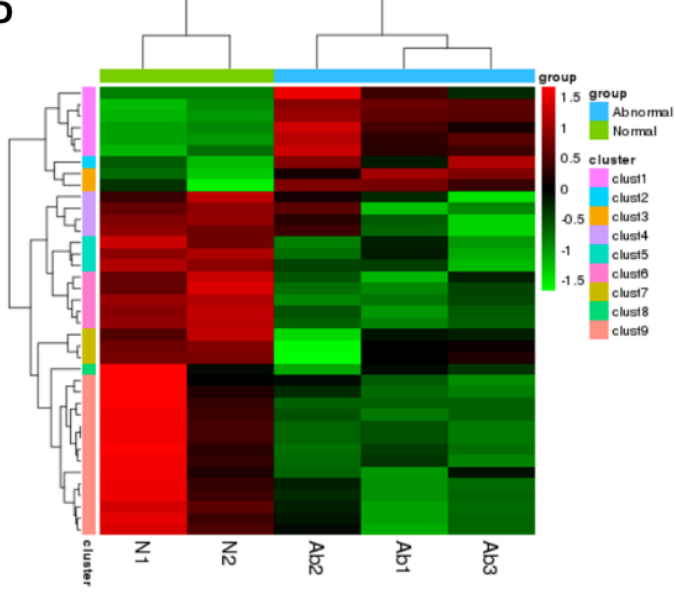

$\mathbf{F}$

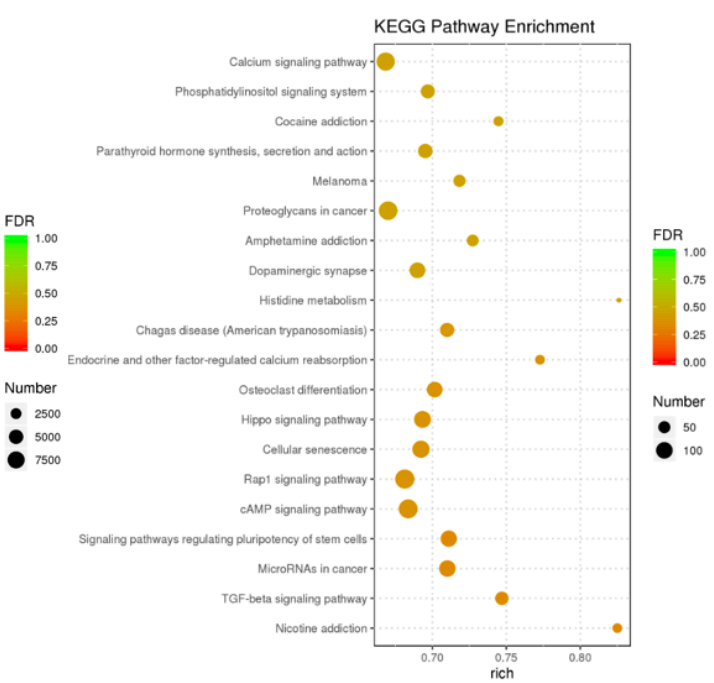

Figure 2. miRNA-Seq analysis for DEGs in control and atrial fibrillation groups. A) PCA analysis of samples from non-AF Ctrl and AF patient samples. B) Average number of miRs analyzed from patients and ctrl group in miRNA-seq. C) Volcano plot shows significantly upregulated (red), downregulated (blue), and non-significant miRs (grey). Significant miRs are selected based on $\log 2 \mathrm{FC}>1$ and $p<0.05$. D) Heat-map matrix shows clustering of differentially expressed miRs between Ctrl and AF groups. E) GO and F) KEGG pathway enrichment analysis of top deregulated pathways. 
A

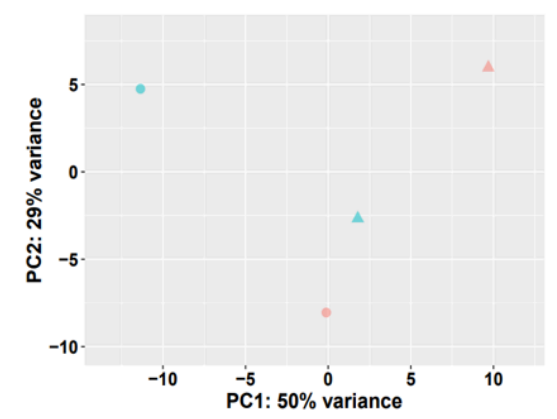

C

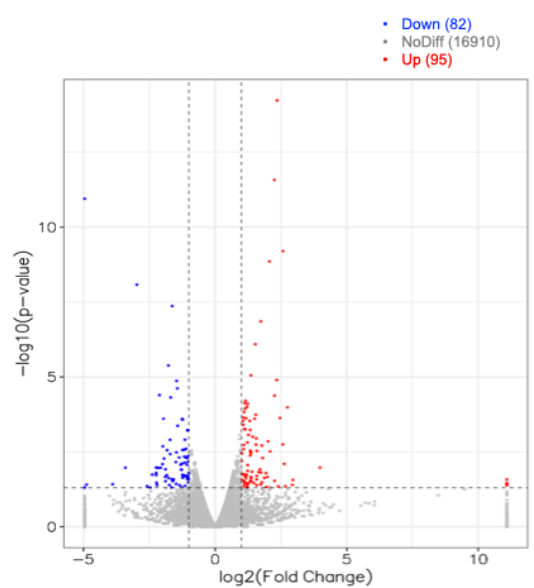

E

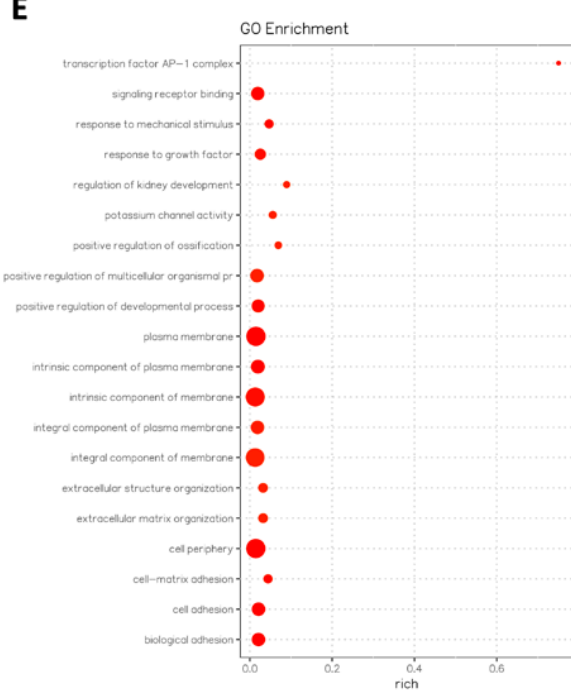

B

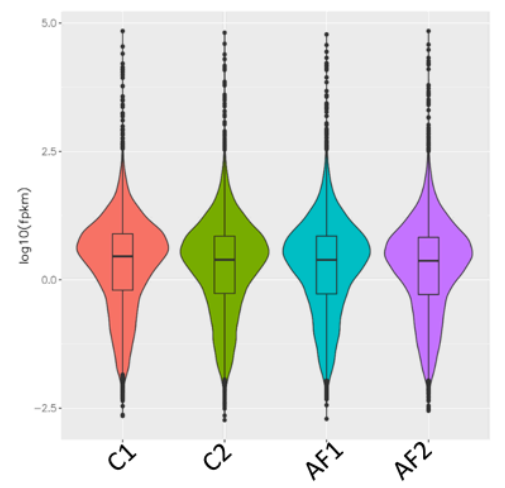

D

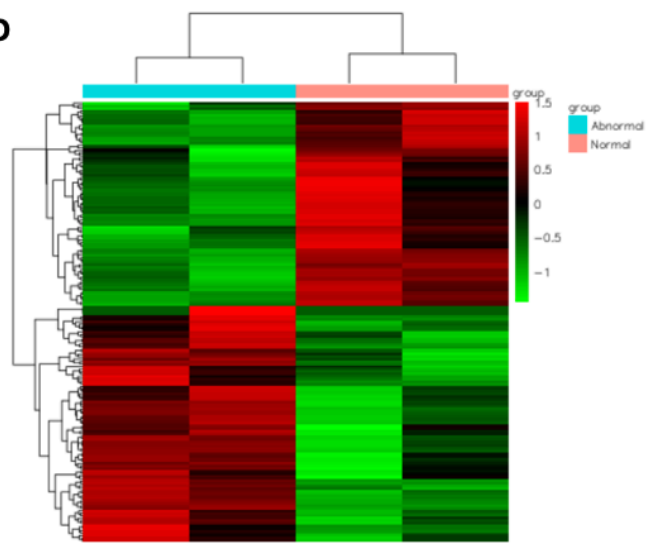

F

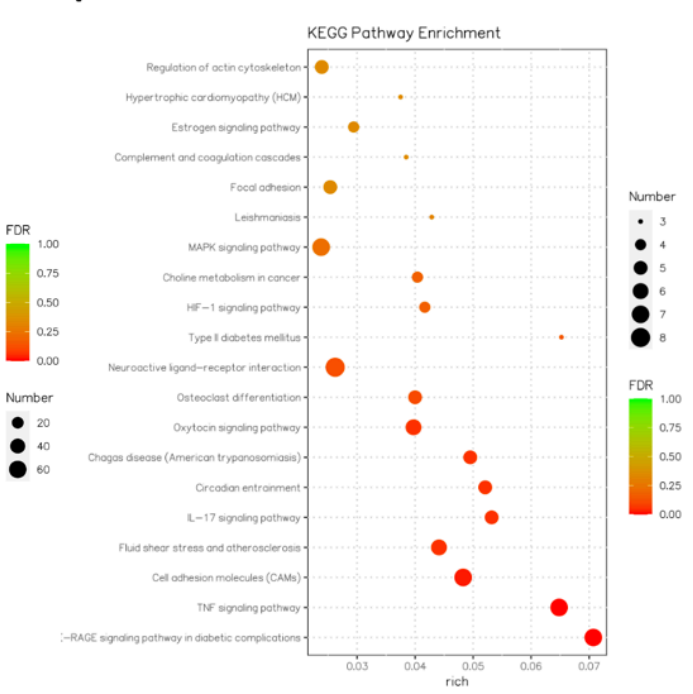

Figure 3. mRNA-seq analysis for DEGs in non-AF control and AF group. A) PCA analysis of samples from non-AF Ctrl and AF patient samples. B) Average number of genes analyzed from patients and ctrl group in mRNA-seq. C) Volcano plot shows significantly upregulated (red), downregulated (blue), and non-significant (grey) genes. Significant genes are selected based on $\log _{2} \mathrm{FC}>$ 1 and $p<0.05$. D) Heat-map shows clustering of differentially expressed genes between Ctrl and AF groups. E) GO and F) KEGG pathway enrichment analysis of top deregulated pathways. 
Table 1. Top deregulated miRNAs

\begin{tabular}{lll}
\hline miR-ID & log2FoldChange & p-value \\
\hline hsa-miR-302b-3p* & -1.927993099 & $3.52446 \mathrm{E}-05$ \\
hsa-miR-3059-5p* & -2.439099768 & 0.000210321 \\
hsa-miR-302d-3p* & -1.925669264 & 0.000598812 \\
hsa-miR-378d* & -1.1161761 & 0.000863964 \\
hsa-miR-516a-5p* & -2.449280109 & 0.001097802 \\
hsa-miR-302a-5p* & -1.511297201 & 0.001691902 \\
hsa-miR-378i* & -1.038634253 & 0.00180456 \\
hsa-miR-518c-3p* & -3.007366398 & 0.002448495 \\
hsa-miR-516b-5p* & -1.640951948 & 0.002989037 \\
hsa-miR-5708* & -1.848531157 & 0.003210251 \\
hsa-miR-302a-3p* & -1.884247241 & 0.003340718 \\
hsa-miR-378e & -1.41425312 & 0.004785214 \\
hsa-miR-302c-3p & -2.078986595 & 0.005110454 \\
hsa-miR-517a-3p & -2.146095895 & 0.006125071 \\
hsa-miR-517b-3p & -2.146095895 & 0.006125071 \\
hsa-miR-526b-5p & -4.103564723 & 0.00733209 \\
hsa-miR-371a-3p & -1.330621285 & 0.01025266 \\
hsa-miR-520g-3p & -1.89787358 & 0.01062743 \\
hsa-miR-585-3p & -1.925975099 & 0.01506384 \\
hsa-miR-372-3p & -1.232670652 & 0.015762249 \\
hsa-miR-4662a-5p & -1.128104683 & 0.016532088 \\
hsa-miR-378h & -1.380015786 & 0.018242992 \\
hsa-miR-520c-3p & -1.84779246 & 0.028579751 \\
hsa-miR-523-3p & -2.295223982 & 0.039597527 \\
hsa-miR-520a-3p & -1.640624273 & 0.03967688 \\
hsa-miR-519a-5p & -2.207467024 & 0.04264619 \\
hsa-miR-1323 & -2.430852745 & 0.045529514 \\
hsa-miR-520f-3p & -1.351910815 & 0.046170691 \\
hsa-miR-520b-3p & -2.29949029 & 0.047158202 \\
\hline hsa-miR-146b-5p* & 2.347289373 & $7.56934 \mathrm{E}-09$ \\
hsa-miR-146b-3p* & 2.289327335 & $2.21466 \mathrm{E}-07$ \\
hsa-miR-155-5p* & 1.274620071 & 0.00071305 \\
hsa-miR-3690* & 1.641664997 & 0.004215924 \\
hsa-miR-187-5p* & 1.844437031 & 0.011552148 \\
hsa-miR-187-3p* & 1.028719714 & 0.013972754 \\
hsa-miR-592* & 3.091036819 & 0.023513587 \\
hsa-miR-212-3p* & 1.148408853 & 0.024049925 \\
hsa-miR-549a-3p* & 2.91790048 & 0.026012753 \\
\hline Differentially expressed microRNAs with log2FC<-1 or \\
log2FC $>1$ and statistical value of p $<0.05$. \\
*Top selected miRs for constructing a regulatory network \\
of miR-gene pairs. & & \\
& &
\end{tabular}

2.2. Network Analysis Downregulated miRs and Upregulated Genes and Selection of Candidate Pairs

To find out affected gene sets among differentially upregulated genes, we run gene-set enrichment analysis (GSEA) and showed that 12 pathways were significantly enriched with differentially upregulated genes (Figure 4A). Among these pathways, TNF- $\alpha$ inflammatory signaling was the top affected pathway, which is usually upregulated in AF and is a sign of ongoing inflammatory process [25]. Also, other hallmark pathways, including p53 signaling and epithelial-to-mesenchymal transition (EMT), were highly enriched (Figure 4A-B). To find out miR-gene pairs, we searched predicted targets of top-11 differentially downregulated miRs (Table-1) in miRDB database (www.mirdb.org) and constructed interaction network with negatively correlated genes in RNAseq by using Cytoscape 3.8.2 [26]. To prioritize genes with relatively high enrichment in RNAseq, we included only top-50 enriched genes from GSEA (Figure 4C) and overlaid them with predicted targets of top-11 differentially downregulated miRs, which resulted with 9 genes (FAM72A, KYAT1, LRRC38, SDC1, PTCHD4, TYW1B, FCER2, SELE, FBXL16) (Figure 4D), 6 miRs (miR-3059-5p, miR-302a-5p, miR-516b-5p, miR-302b-3p, miR-302d-3p, miR-302a-3p), and 11 interactions between them (Figure 4E; Table-2). GO terms of these 9 identified genes showed no shared functional annotation (www.david.ncifcrf.org), except 6 of them (LRRC38, SDC-1, PTCHD4, TYW1B, FCER2, SELE) being plasma membrane-associated proteins (Data not shown). Overall, these highly enriched genes and their corresponding miRs are potential candidates that may involve in AF pathogenesis. 
A
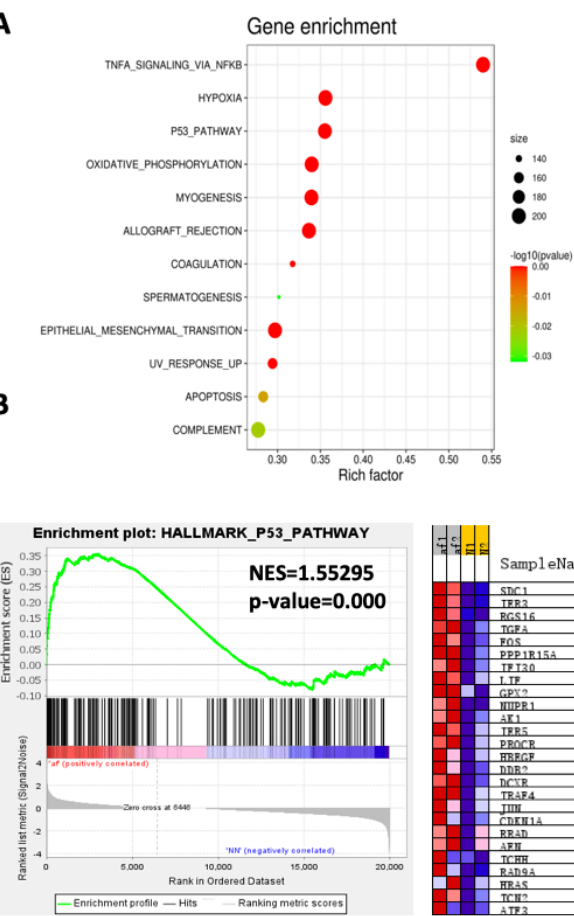

Enrichment plot:

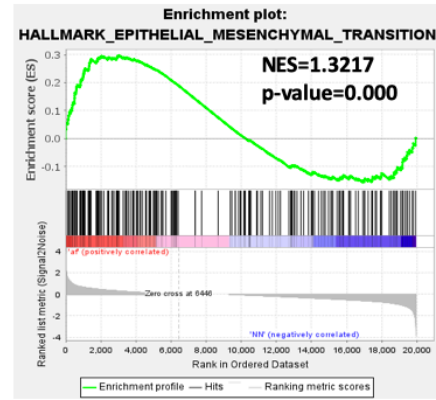

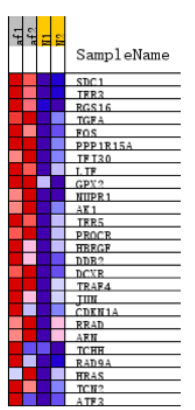

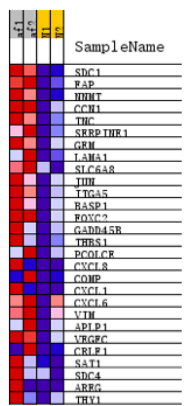

C
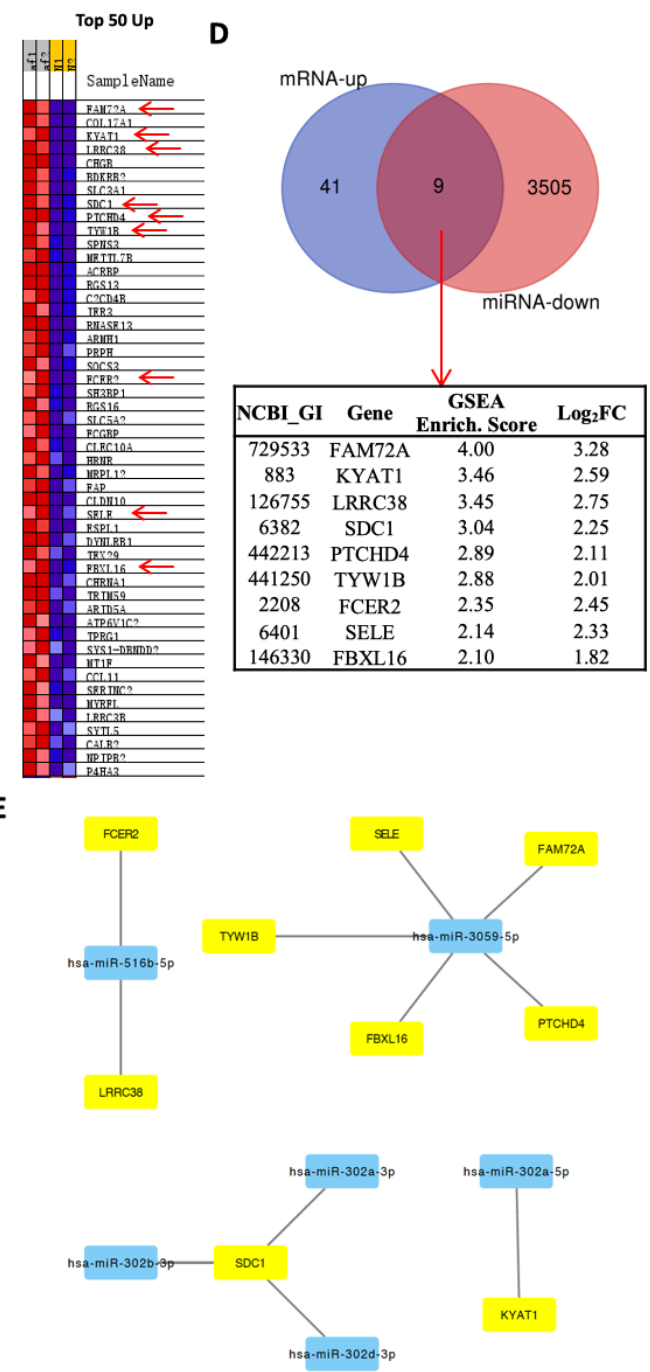

Figure 4. Analysis of upregulated genes and selection of candidate miR-mRNA pairs. A) Gene-set enrichment analysis (GSEA) of upregulated genes. B) Enrichment plots of some of the top enriched pathways. C) Heat-map showing Top-50 positively enriched genes in GSEA based on enrichment score. D) Venn diagram shows overlapping genes between Top50 positively enriched genes in GSEA (blue) and all predicted targets of Top-11 downregulated miRs in RNA-seq (red). 9 overlapping genes between groups were selected as potential candidates and their enrichment scores and $\log _{2}$ FoldChanges listed in the table. E) Interaction network of positively enriched genes in GSEA and their negatively correlated microRNAs.

\begin{tabular}{cccccc}
\multicolumn{5}{c}{ Table 2. Overlapping genes between predicted targets of top 11 downregulated miRs and top 50} \\
positively enriched genes in GSEA
\end{tabular}


2.3. Network Analysis of Upregulated miRs and Downregulated Genes and Selection of Candidate Pairs

Our gene-set enrichment analysis (GSEA) for downregulated genes showed that 5 pathways were enriched among significantly downregulated genes (Figure 5A). Among these pathways, Notch and Hedgehog signaling were the top affected pathways (Figure 5B). To find out potential miR-gene pairs, we overlaid predicted targets of top- 9 differentially upregulated miRs in Table-1 (www.mirdb.org) and top-50 negatively enriched genes in GSEA (Figure 5C). 6 common genes were identified as potential candidates (Figure 5D). Subsequently, we constructed an interaction network of negatively correlated pairs in Cytoscape 3.8.2 (Figure 5E). Our analysis identified 6 genes (FERMT1, SLC36A2, GPM6B, CCNI2, MCTP2, GUCY1A2), 7 miRs (miR-146b-5p, miR-155-5p, miR-3690, miR-187-5p, miR-187-3p, miR-592, and miR-549a-3p), and 8 interactions between them (Figure 5E; Table-3). Overall, these highly enriched genes and their corresponding miRs may involve in pathogenesis of AF.

A<smiles>[10BH]</smiles>

B
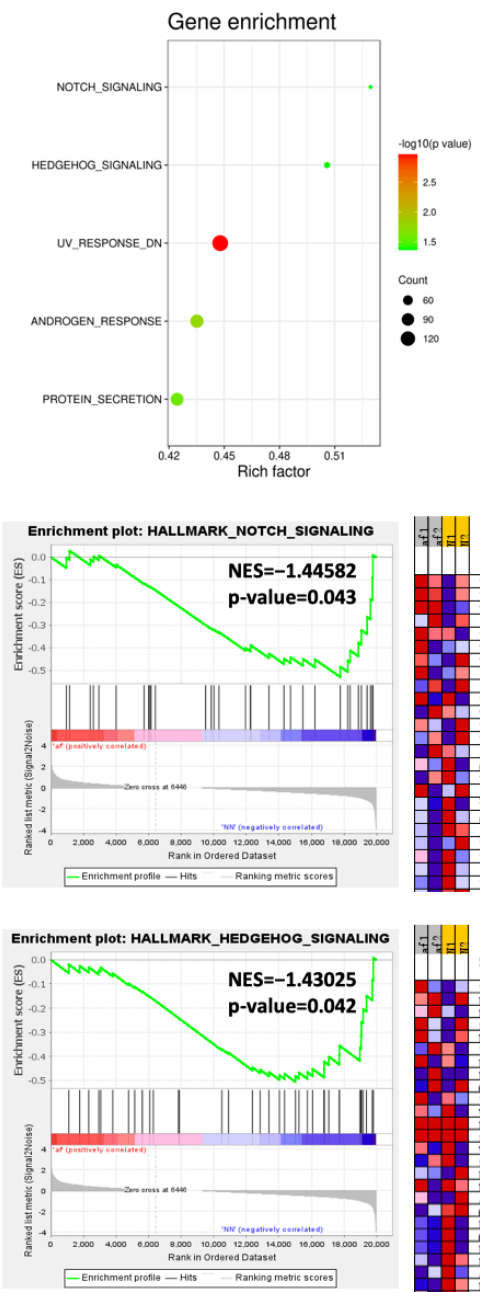

C

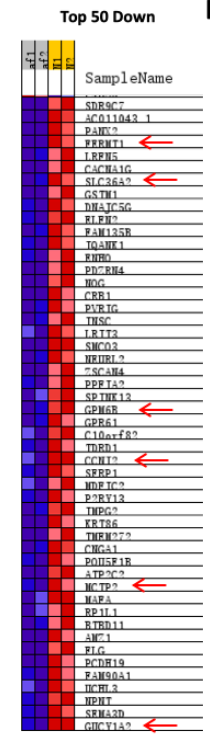

D

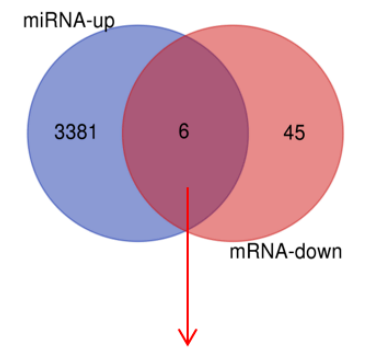

\begin{tabular}{|cccc|}
\hline NCBI_GI & Gene & $\begin{array}{c}\text { GSEA } \\
\text { Enrich. Score }\end{array}$ & Log 2 FC \\
\hline 55612 & FERMT1 & -3.13 & -2.00 \\
153201 & SLC36A2 & -2.98 & -3.52 \\
2824 & GPM6B & -2.45 & -1.46 \\
645121 & CCNI2 & -2.40 & -1.90 \\
55784 & MCTP2 & -2.32 & -2.12 \\
2977 & GUCY1A2 & -2.21 & -1.28 \\
\hline
\end{tabular}

E
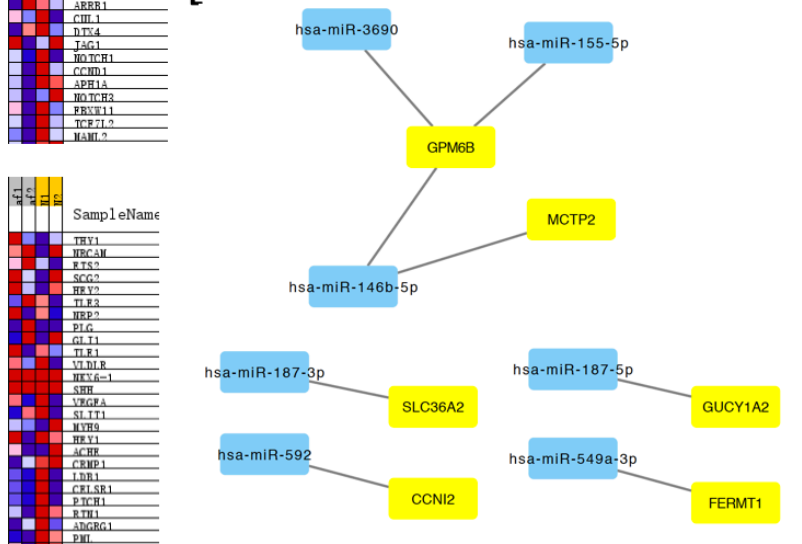

Figure 5. Analysis of downregulated genes and selection of candidate miR-mRNA pairs. A) Gene-set enrichment analysis (GSEA) of downregulated genes. B) Enrichment plots of some of the top enriched pathways. C) Heat-map showing Top-50 negatively enriched genes in GSEA based on enrichment score. D) Venn diagram shows overlapping genes between Top50 negatively enriched genes in GSEA (blue) and all predicted targets of Top-9 upregulated miRs in RNA-seq dataset (red). 6 overlapping genes between groups were selected as potential candidates and their enrichment scores and $\log _{2}$ FoldChanges listed in the table. E) Interaction network of negatively enriched genes in GSEA and their negatively correlated microRNAs. 


\begin{tabular}{|c|c|c|c|c|c|}
\hline Gene Name & NCBI_GI & $\begin{array}{c}\text { Rank among Top } 50 \\
\text { Downregulated } \\
\text { mRNAs }\end{array}$ & miRNA ID & $\begin{array}{l}\text { Rank among } \\
\text { Upregulated } \\
\text { miRs (out of 9) }\end{array}$ & $\begin{array}{c}\text { miRdb } \\
\text { Target Score } \\
\text { (out of 100) }\end{array}$ \\
\hline FERMT1 & 55612 & 4 & hsa-miR-549a-3p & 9 & 67 \\
\hline SLC36A2 & 153201 & 7 & hsa-miR-187-3p & 6 & 57 \\
\hline \multirow{3}{*}{ GPM6B } & \multirow{3}{*}{2824} & \multirow{3}{*}{25} & hsa-miR-146b-5p & 1 & 86 \\
\hline & & & hsa-miR-155-5p & 3 & 82 \\
\hline & & & hsa-miR-3690 & 4 & 80 \\
\hline $\mathrm{CCNI2}$ & 645121 & 29 & hsa-miR-592 & 7 & 57 \\
\hline MCTP2 & 55784 & 39 & hsa-miR-146b-5p & 1 & 78 \\
\hline GUCY1A2 & 2977 & 50 & hsa-miR-187-5p & 5 & 76 \\
\hline
\end{tabular}

\subsection{Selecting a Candidate Gene to Experimental Validate Its Involvement in AF}

We further analyzed genes in GSEA analysis to find out what pathways were highly affected by top-enriched genes in GSEA. Protein-protein interaction (PPI) network was constructed for the genes with enrichment score of ES $>1.5$ (Figure S1A) or ES $<-1.5$ (Figure S2A). Genes with at least one interaction and confidence score $>50$ were included in the analysis. PPI network of positively enriched genes showed that SDC-1 and SELE, some of the candidates in Figure 4E, were in direct or indirect interactions with a group of AF-associated genes (Figure S1A-B). p53 and TNF- $\alpha$ hallmark pathways were highly enriched by the genes in PPI network (Figure 4B and Figure S1C). Also, canonical pathway analysis (www.gsea-msigdb.org) showed significant enrichment of ATF2, AP-1, FRA, and SYNDECAN-1 pathways (Figure S1D). Indeed, SDC-1 was a shared gene in both p53 and EMT signaling (Figure 4B and Figure S1C) as well as in SYDECAN-1 pathway (Figure S1D), and thus stands out as a promising candidate possibly involving in structural remodeling of atria. Besides, GO terms found SDC-1 involving in top significant terms (Figure S1E-G). As for negatively enriched genes, we found no significant interaction with AF-associated genes (Figure S2A-B). However, GPM6B, one of the candidate genes in Figure 5E, was in a major interaction network with neuronal genes (Figure S2A-B). GPM6B was not found in any significantly enriched hallmark pathways (Figure S2C and S2D), despite GPM6B being a neuron-specific gene. However, neuron-related gene sets were highly enriched among genes from PPI network. Also, neuron-related GO terms were consistently enriched and showed strong association with GPM6B (Figure S2E). Thus, GPM6B was a considerably good candidate possibly involving in neuronal remodeling of atria.

As SDC-1 was in interaction with AF-associated genes (Figure S1A) and it was highly enriched in p53, EMT, and SYNDECAN_1 signaling pathways, we decided to experimentally validate SDC-1 and its corresponding miRs. MiR-302 family had a very high prediction score (96 out of 100) targeting SDC-1 gene (Table-2). We initially checked gene and protein expression levels of SDC- 1 in human atrial tissue samples. AF patients had significantly elevated levels of SDC- 1 mRNA $(2.12 \pm 0.13, n=6$ vs. $1.074 \pm 0.18, n=6$ in Ctrl) (Figure 6A) and protein expression ( $0.91 \pm 0.07, \mathrm{n}=3$ vs. $0.42 \pm 0.04, \mathrm{n}=3$ in Ctrl) (Figure 6B). Consistently, expression levels of miR-302a-3p (0.18 $\pm 0.05, \mathrm{n}=6$ vs. $1.09 \pm$ $0.20, \mathrm{n}=6$ in Ctrl), miR-302b-3p $(0.20 \pm 0.03, \mathrm{n}=6$ vs. $1.08 \pm 0.20, \mathrm{n}=6$ in Ctrl $)$, and miR-302d-3p $(0.14 \pm 0.03, n=6$ vs. $1.12 \pm 0.22, n=6$ in Ctrl $)$ were significantly reduced in AF tissues (Figure 6C), showing a negative correlation with SDC-1. miR-302-3p family has a conserved seed sequence and thus have high overlap of their target genes (Figure 6D). To evaluate whether they all target SDC-1 and reduce mRNA levels, we transfected 293T cells with either of all three isotypes, which were found among top significantly downregulated miRs in our miRseq (Table-1), and showed efficient overexpression levels (miR-302a: $17.03 \pm 2.36, \mathrm{n}=6$; miR-302b: $127.1 \pm 40.3, \mathrm{n}=6$; miR-302d: $148.8 \pm 5.81, \mathrm{n}=4$; vs. Ctrl: $1.03 \pm 0.15, \mathrm{n}=4$ ) (Figure 6E). However, only miR-302b-3p showed a significant reduction in SDC- 1 levels ( $1.08 \pm 0.23, \mathrm{n}=4$ vs. $0.43 \pm 0.04, \mathrm{n}=6$ in Ctrl) (Figure 6F). These all together suggested that miR-302b-3p/SDC-1 axis may have a role in AF pathogenesis. 
A

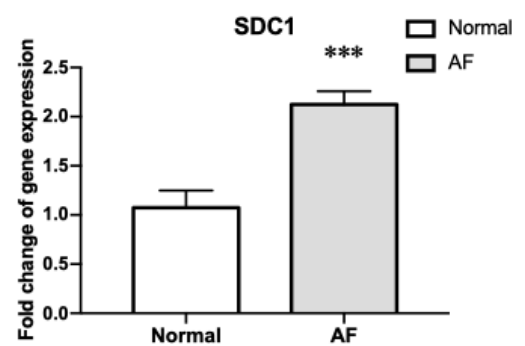

B

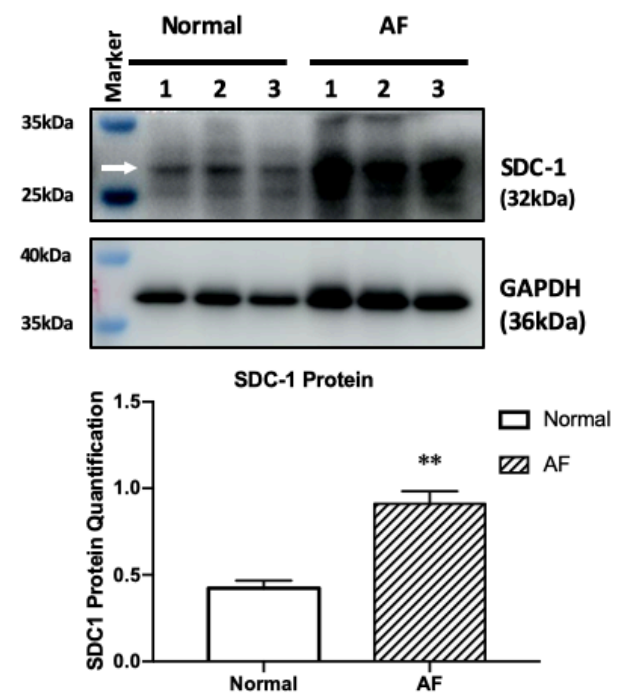

C

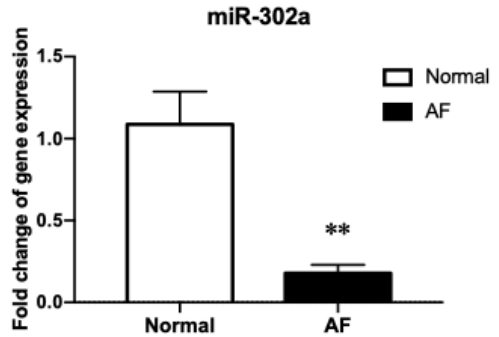

miR-302b

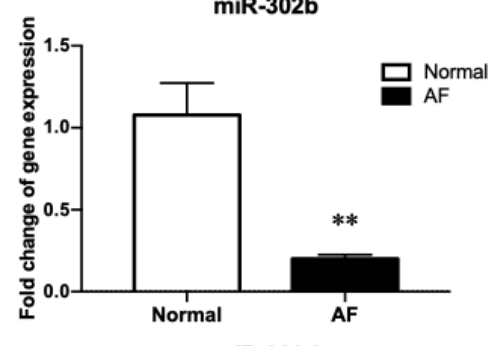

miR-302d

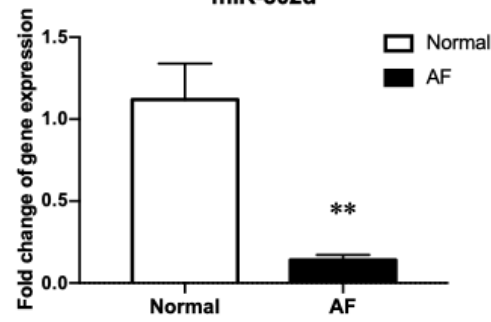

D

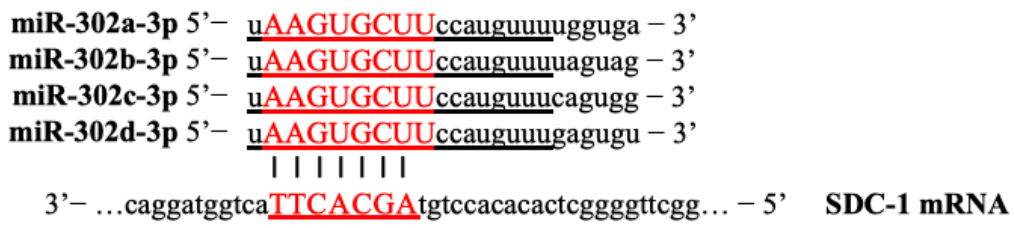

E

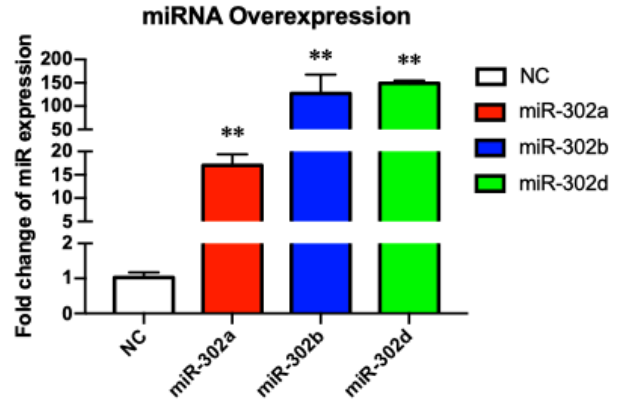

$\mathbf{F}$

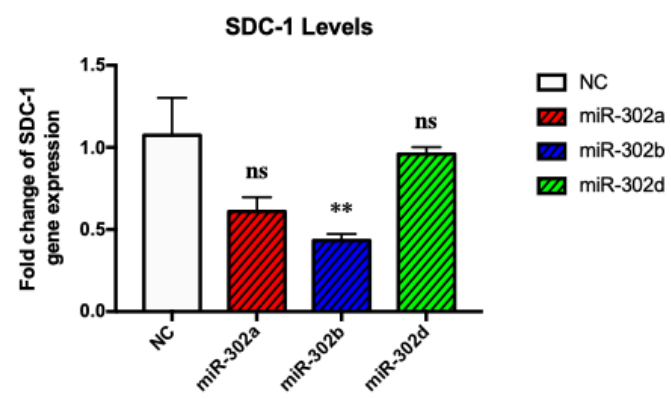

Figure 6. Validation of a selected miR-gene pair in human tissue and cells. A) SDC-1 mRNA and B) protein expression A-B) levels in Ctrl vs. AF atrial tissue samples. C) Expression levels of miR-302 family in atrial tissue samples of Ctrl vs. AF groups. D) Conserved sequences of miR-302 family. Underlined sequences indicate conserved nucleotides including their seed sequences. Seed sequences were highlighted in red capital letters. E) Transfection of Ctrl. vs. miR-302 mimics to 293T cells. F) qRT-PCR shows SDC-1 gene expression levels after transfection with miR mimics. ** $p<0.01$ or ${ }^{* * *} p<0.005$.

\section{Discussion}

Non-coding RNAs play critical roles in development and pathogenesis of AF. Recent studies have predicted novel genes and non-coding RNAs by RNAseq of transcriptome in patient-derived atrial samples [27-31]. Here in our RNAseq data, we performed similar analysis techniques to profile differential expression of miRNAs ( 9 up and 30 down) and mRNAs (95 up and 82 down). To be able to identify potential miR-mRNA 
pairs, we followed a different strategy by further analyzing enriched genes in GSEA, which are highly enriched among all sequenced mRNAs, and overlaying them with differentially expressed miRs. Briefly, top-50 positively or negatively enriched genes (Figure $4 \mathrm{D}$ and 5D, respectively) were overlaid with predicted targets of top differentially expressed miRs (Figure 4E and 5E). Interaction network of common genes between two groups and their corresponding miRs was constructed by Cytoscape. Eventually, we identified 15 genes and $11 \mathrm{miRs}$ in total that are negatively correlated in expression (Table 2 and Table 3), which will be discussed below in detail.

We linked 9 positively enriched genes (Figure 4D) and 6 negatively regulated miRs (Figure 4E; Table 2). Among these genes, TYW1B, FCER2, SELE, and SDC-1 had some degree of connections with cardiovascular diseases including AF. TYW1B is associated with triglyceride metabolism in heart disease [32,33]. GWAS studies found novel variants of genes including TYW1B that are associated with altered triglyceride levels in heart disease. FCER2, a B-cell specific antigen involving in inflammatory response, is associated with cardiovascular disease [34]. SELE (Selectin E) is an endothelial cell-specific surface protein involved in leukocyte attachment to endothelial cells. Previously, genetic variants of SELE were strongly associated with cardiovascular disease [35-38]. Interestingly, increased levels of soluble endothelial markers, including SELE, and endothelial dysfunction have been co-observed in AF patients [39,40]. Lastly, SDC-1 (Syndecan-1) is a transmembrane heparan sulfate proteoglycan, which plays critical roles in cell-cell adhesion and intercellular communication [41,42]. SDC-1 has previously not been associated with AF although it has been studied in various different disease settings including heart disease [43-48]. In contrary, FAM72A, KYAT1, LRRC38, and PTCHD4 had little or no known associations with cardiovascular diseases so far although they are moderately expressed in heart and may have unidentified functions in AF. We also linked 6 negatively enriched genes (Figure 5D) and 7 upregulated miRs (Figure 5E; Table 3). Among these, expression of SLC36A2 in epicardial adipose tissue is linked with increased risk of coronary artery disease [49]. GPM6B regulates smooth muscle cell differentiation through controlling TGF- $\beta$-Smad $2 / 3$ axis via direct interaction with T $\beta$ RI (TGF- $\beta$ receptor 1 ) [50]. Indeed, fibrosis, one of the hallmarks of AF, is widely induced by TGF- $\beta$ signaling, and smooth muscle abnormalities were coexistent with fibrosis in AF patients [51]. MCTP2 is an essential gene required for proper development of left ventricular outflow tract, and genetic mutations in this gene result with abnormal development of outflow tracts [52-54]. Also, genetic variants of MCTP2 have been associated with heart rhythm increase [55,56]. Lastly, a GUCY1A2 single-nucleotide polymorphism was associated with hypertension and is useful in detection of patients predisposed to hypertension [57]. In contrary, FERMT1 and CCNI2 have no direct link with any form of cardiovascular diseases so far although they may have some unknown functions in AF. Altogether, given that some of these genes are already associated with cardiovascular diseases, they may involve in pathogenesis of AF along with their corresponding miRs. Among these, SDC-1 stands out as a strong candidate based on our analyses, and its potential involvement in heart disease and AF will be extensively discussed below.

SDC-1 was reported to function in heart failure by promoting fibrosis through TGF- $\beta / S m a d 2[23,58,59]$ or p38/MAPK [24] pathway. Indeed, TGF- $\beta$ was among the highly impacted signaling pathways in KEGG pathway analysis (Figure 2F). Also, our PPI network showed indirect interaction of SDC-1 with TGFA and a group of AF-associated genes (Figure S1A and S1B). On the other hand, cell adhesion molecules, including SDC-1, were highly enriched in KEGG pathway analysis of DEGs (Figure 3F). Consistently, PPI network of positively enriched genes in GSEA found SYNDECAN-1 pathway as one of the top enriched pathways (Figure S1D). Besides, our data found that SDC-1 was the top enriched gene in both p53 and EMT hallmark pathways, which are among the top-10 significantly enriched pathways in GSEA of DEGs (Figure 4A) and in GSEA of PPI network (Figure S1C). Both p53 signaling [60,61] and EMT [62,63] are involved in various aspects of heart disease, including AF. More specifically, p53 signaling 
was shown to be linked with the premature senescence of atrial fibroblasts in $\mathrm{AF}$, which results in progressive increase of ECM accumulation and perivascular fibrosis $[64,65]$ and is commonly observed in aging individuals [66]. On the other hand, it has been known that EMT is regulated by TGF- $\beta$ in atrial fibroblasts and its effect is further strengthened under proinflammatory TNF- $\alpha$ signaling in AF [67]. To ensure negative correlation between SDC- 1 and miR-302 family in vitro, we checked gene expression of SDC-1 and miRs in atrial tissue samples and validated our RNAseq results (Figure $6 \mathrm{~A}-\mathrm{C}$ ). To validate targeting of SDC- 1 by its potential regulator, miR-302-3p family (Table-2), we transfected 293T cells with mimics of either of three isotypes, which are all significantly downregulated in our RNAseq (Table-1), and validated that miR-302b-3p regulates SDC-1 expression in vitro (Figure 6D-E). Indeed, some members of miR-302 family has been previously shown to target SDC-1 transcripts [68,69], supporting our findings. Collectively, miR-302b-3p/SDC-1 axis may involve in AF through regulating atrial fibrosis, which is connected with profibrotic signaling by TGF- $\beta$, p53, and EMT.

Although pathological remodeling of non-cardiomyocytes alone can induce arrhythmia responses in human [70] and SDC-1 is highly expressed in cardiac fibroblasts $[23,58,71]$, it needs validation to prove whether SDC- 1 function in AF is mediated through fibroblasts. Therefore, further studies on cellular distribution of SDC-1 in atrial heart tissue may shed light on its mechanism in AF. As both miR-302 family and SDC-1 have conserved sequences and function between human and mouse, mouse models may help elucidate molecular mechanisms of miR-302b-3p/SDC-1 axis in AF pathogenesis. Indeed, disease models with iPSC-derived fibroblasts [72] or CMs [73], which are physiologically more similar to native human cells, also offer reliable platform for investigation of AF mechanisms as the tissue resources from patients is limited for extensive mechanistic research [73,74]. Experimental validations of miR-302b-3p/SDC-1 function and mechanism may help generate therapeutic approaches targeting this axis toward the treatment of AF patients.

Our study had some limitations. Some important genes or miRs might have been missed out due to our small sample size. Regardless of this issue, we had sufficient miR $\left(>42 \times 10^{6}\right)$ and mRNA $\left(49 \times 10^{6}\right)$ reads detected in our RNAseq data, and could successfully detect differential expression of some of the previously identified miRs and genes. For example, miR-146b-5p - the most significantly upregulated miR in our miRseq (Table 1) - was previously found to be upregulated in AF, regulating structural $[75,76]$ and electrical remodeling of atria [77]. Besides, miR-302 family was found deregulated in AF in some studies [78,79]. Also, among the significantly upregulated genes, SELE, as an example, has been previously associated with AF [80]. 


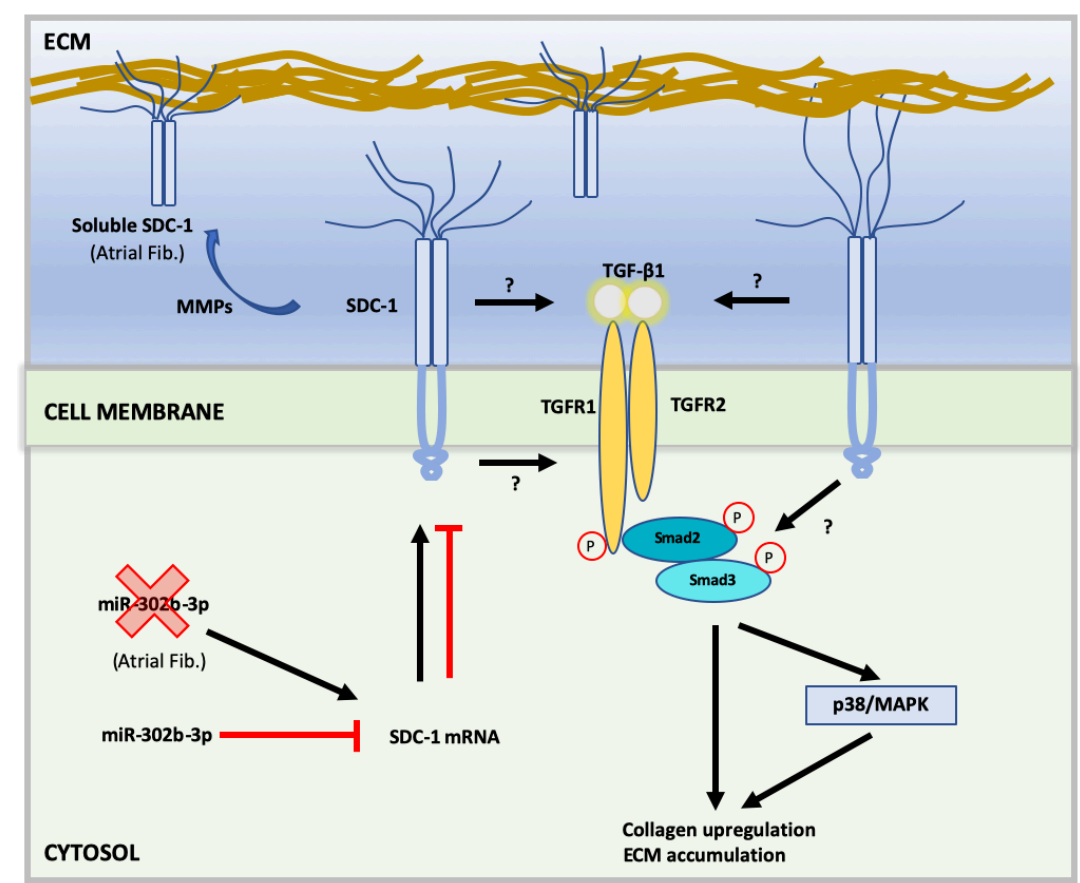

Figure 7. Proposed mechanism of SDC-1 in fibrotic remodeling of AF. Decreased levels of miR-302-3p family will result in increased expression of Syndecan-1 in atrial cardiac fibroblasts, which will stimulate TGF- $\beta / S \operatorname{mad} 2-$ mediated atrial fibrosis.

\section{Materials and Methods}

\subsection{Preparation of RNA Samples and RNA Sequencing}

Total RNA was extracted from atrial tissue samples using Trizol Reagent (Invitrogen) and RNA quality was determined by NanoDrop (Thermoscientific). Sequencing libraries were generated from $3 \mu \mathrm{g}$ RNA using the TruSeq RNA Sample Preparation Kit (Illumina Inc.). For mRNAseq, mRNA was purified from total RNA samples using magnetic beads attached to poly-T oligos. Fragmentation was performed in an Illumina proprietary fragmentation bugger using divalent cations under high temperature. SuperScript II was used to synthesize first strand cDNA with random oligonucleotides. Subsequently, second strand was synthesized by DNA Polymerase I and RNase H. Remaining overhangs were blunted by an exonuclease/polymerase and then enzymes were removed. Following 3'-end biotinylation, Illumina PE adapter oligos were ligated to DNA fragments to prepare for hybridization. Library fragments were purified by AMPure XP system (Beckman Coulter, Beverly, CA, USA) to select cDNA fragments with preferred $300 \mathrm{bp}$ in length. DNA fragments with adapter fragments on both ends were selectively enriched in a 15-cycle PCR reaction with Illumina PCR Primer Cocktail. Resulted products were then purified (AM Pure XP system) and quantified using a DNA quantification assay on Bioanalyzer 2100 system (Agilent). Finally, cDNA library was sequenced by Shanghai Personal Biotech Cp. Ltd. (Shanghai, China) on a Hiseq X platform (Illumina).

\subsection{RNAseq Data Analyses}

Differentially expressed gene (DEG) analysis was performed using DESeq (Version 1.18.0). Nominal p-values were adjusted by the false data discovery rate (FDR) criterion as needed. Genes with log2FoldChange $>1$ (or FoldChange $>1.5$ ) and statistical value of $p$ $<0.05$ were considered statistically significant changes and demonstrated on heatmap. Significantly deregulated genes were then used for subsequent analyses.

Gene ontology (GO) and Kyoto Encyclopedia of Eenes and Eenomes (KEGG) pathway analyses were performed for differentially expressed miRs and genes using DAVID database. Hallmark gene enrichment analysis was run in GSEA 
(www.gsea-msigdb.org) to find out deregulated signaling pathways. Cross-tabulation analysis (Venn analysis) was performed to obtain the expression of predicted AF-related genes. The results of the raw signal analysis were then further validated by searching the literature and in vitro experiments. Cytoscape (V3.6) was used to demonstrate the interaction network of miRNAs and target genes. Venn diagrams showing overlay of different gene sets were generated by Venny v2.1.

Protein-protein-interaction (PPI) network was constructed for genes, which are in GSEA analysis of differentially expressed genes. Genes with positive enrichment score of ES $>1.5$ or negative enrichment score of ES<-1.5 were selected for PPI network in Cytoscape. Genes with at least one interaction and confidence score of $>50$ were shown in PPI network. Positively or negatively enriched genes in PPI network with the set criterion were searched through molecular signatures database (MSigDB v7.4) for GO and Canonical pathway analyses. Overlay of genes in PPI network and AF-related genes in our RNAseq data were visualized by Venny2.1. AF-related genes were obtained from gene-disease association database (www.disgenet.org) by searching the term "Atrial Fibrillation; CUI: C0004238".

\subsection{Gene Expression Analysis by qRT-PCR}

The sequencing results were validated with quantitative real-time PCR method. At the tissue level, total RNA was extracted from the right atrial appendage of AF and non-AF patients. The total RNA of cells and tissues were extracted, and microRNAs and mRNA were reversed transcribed to cDNA using the Reverse Transcription SparkJade MicroRNA/mRNA Reverse Transcription Kit that is the method of adding a tail (Cat\#. AG0501/AG0304, SparkJade, Qingdao, China)) according to manufacturer's instructions. qRT-PCR was performed using SYBR Green Master Mix (SparkJade, Qingdao, China) on a Bio-Rad iCycler.[81]. Primer sequences for gene expression analysis are listed as followed.

H-SDC1-Forward: ACTCATCTGGCCTCAACGAC

H-SDC1-Reverse: GTGTGGGGAGTGTGAAGGTC

H-GAPDH-Forward: GCACCGTCAAGGCTGAGAAC

H-GAPDH-Reverse: TGGTGAAGACGCCAGTGGA

Hsa-miR-302d-3p Forward: ACUUUAACAUGGAGGCACUUGC

Hsa-miR-302b-3p Forward: UAAGUGCUUCCAUGUUUUAGUAG

Hsa-miR-302a-3p Forward: UAAGUGCUUCCAUGUUUUGGUGA

Hsa-U6 Forward: Purchased from Beijing Tiangen Biochemical Tech., Co., LTD

miRNA Reverse: Provided in the qPCR kit (SparkJade, Qingdao, China)

\subsection{Protein Expression Analysis with Western Blot}

For total protein collection, tissues and cells were harvested following homogenization in RIPA lysis buffer with phosphatase and protease inhibitors, followed by sonication for 10s. Protein concentration was measured by Biyuntian BCA protein kit (Cat\# P0012, Beyotime), and protein samples were denatured in 5x loading buffer (Cat\# P0015L, Beyotime) by boiling for 10 minutes at $95^{\circ} \mathrm{C}$. Lysates were run in $10 \%$ SDS-PAGE gels. Proteins were transferred to PVDF membranes, followed by 1 hour blocking with 10\% milk solution at room temperature. Primary antibodies against the following proteins were used: human-GAPDH (1:1000, Cat\#4478, Cell Signaling Tech., MA, USA) and human-SDC1 (1:1000, Cat\# EPR6456, Abcam) overnight at 4 C. Membranes were washed three times and incubated with HRP-conjugated anti-rabbit secondary antibodies (1:3000, Cat\#7074, Cell Signaling Tech., MA, USA) for 1-2 hours at room temperature. Signal was detected using Clarity Western ECL Substrate (Bio-Rad).

\subsection{Statistical Analyses}

For RNAseq data analysis, we used the hypergeometric distribution method. For GO terms and KEGG pathway analyses, nominal $p$ values were used or FDR approach was applied to adjust p-values as needed. Two-tailed unpaired t-test was used for statis- 
tical analyses of qRT-PCR and Western blot results in Prism 7. For multiple groups, we used one-way ANOVA. All data were shown as mean \pm SEM, and $p<0.05$ was considered statistically significant change.

\section{Conclusions}

In conclusion, RNAseq of atrial samples from AF patients identified differentially expressed small non-coding and coding transcripts. Our network analysis revealed negatively correlated pairs of miRNAs and mRNAs among the top deregulated transcripts. We found that the miR-302b-3p/SDC-1 axis is a potential candidate that may function in development or sustenance of $\mathrm{AF}$ in patients through modulation of atrial fibrosis via TGF signaling. In the future, experimental animal models or human iPSC models may be utilized to study mechanism of miR-302-3p/SDC-1 axis in pathogenesis of AF, and it may serve as a potential therapeutic target in treatment of AF patients.

Supplementary Materials: All supplementary figures and tables are available online at www.mdpi.com/xxx.

Author Contributions: Conceptualization, R.W. and E.B; methodology, R.W. and E.B; validation, R.W., E.B. and P.S.; formal analysis, R.W. and E.B.; investigation, R.W., E.B., P.S., W.H.; resources, J.Z..; data curation, R.W., E.B., P.S., X.M., Y.W., M.C., X.W., J.C., and S.H.; writing-original draft preparation, E.B.; writing-review and editing, J.Z. and R.W.; visualization, R.W. and E.B.; supervision, J.Z. and E.B.; project administration, J.Z.; funding acquisition, J.Z. All authors have read and agreed to the published version of the manuscript.

Funding: This research was funded by National Natural Science Foundation of China, and Jingquan Zhong was supported by grant number 81970282.

Institutional Review Board Statement: The study was conducted under the guidelines of the Declaration of Helsinki, and the use of human tissue samples was approved by Qilu Hospital of Shandong University Research Ethical Committee (APPROVAL \# KYLL-2021(ZM)-231).

Informed Consent Statement: Human atrial heart tissue samples were obtained from the Tissue Bank of Qilu Hospital of Shandong University from patients undergoing regular surgical operation. Tissues samples were from de-identified patients and all data and results presented in this manuscript do not include any patient details.

Data Availability Statement: All supporting data and materials are available online. RNAseq datasets are available from the corresponding authors upon a reasonable request.

Acknowledgments: We are grateful to Tissue bank at Qilu Hospital in China, supported by Qingdao Key Health Discipline Development Fund, for providing access to patient-derived tissue samples.

Conflicts of Interest: The authors declare no conflict of interest. The funders had no role in the design of the study; in the collection, analyses, or interpretation of data; in the writing of the manuscript, or in the decision to publish the results.

\section{References}

1. Wyndham, C.R. Atrial fibrillation: the most common arrhythmia. Tex Heart Inst J 2000, 27, 257-267.

2. Iwasaki, Y.K.; Nishida, K.; Kato, T.; Nattel, S. Atrial fibrillation pathophysiology: implications for management. Circulation 2011, 124, 2264-2274, doi:10.1161/CIRCULATIONAHA.111.019893.

3. Du, X.; Dong, J.; Ma, C. Is Atrial Fibrillation a Preventable Disease? J Am Coll Cardiol 2017, 69, 1968-1982, doi:10.1016/j.jacc.2017.02.020.

4. $\quad$ Chugh, S.S.; Havmoeller, R.; Narayanan, K.; Singh, D.; Rienstra, M.; Benjamin, E.J.; Gillum, R.F.; Kim, Y.H.; McAnulty, J.H., Jr.; Zheng, Z.J., et al. Worldwide epidemiology of atrial fibrillation: a Global Burden of Disease 2010 Study. Circulation 2014, 129, 837-847, doi:10.1161/CIRCULATIONAHA.113.005119.

5. $\quad$ Kotecha, D.; Calvert, M.; Deeks, J.J.; Griffith, M.; Kirchhof, P.; Lip, G.Y.; Mehta, S.; Slinn, G.; Stanbury, M.; Steeds, R.P., et al. A review of rate control in atrial fibrillation, and the rationale and protocol for the RATE-AF trial. BMJ Open 2017, 7, e015099, doi:10.1136/bmjopen-2016-015099. 
6. Kirchhof, P.; Benussi, S.; Kotecha, D.; Ahlsson, A.; Atar, D.; Casadei, B.; Castella, M.; Diener, H.C.; Heidbuchel, H.; Hendriks, J., et al. 2016 ESC Guidelines for the management of atrial fibrillation developed in collaboration with EACTS. Eur J Cardiothorac Surg 2016, 50, e1-e88, doi:10.1093/ejcts/ezw313.

7. Hijazi, Z.; Oldgren, J.; Lindback, J.; Alexander, J.H.; Connolly, S.J.; Eikelboom, J.W.; Ezekowitz, M.D.; Held, C.; Hylek, E.M.; Lopes, R.D., et al. A biomarker-based risk score to predict death in patients with atrial fibrillation: the ABC (age, biomarkers, clinical history) death risk score. Eur Heart J 2018, 39, 477-485, doi:10.1093/eurheartj/ehx584.

8. Schnabel, R.B.; Yin, X.; Gona, P.; Larson, M.G.; Beiser, A.S.; McManus, D.D.; Newton-Cheh, C.; Lubitz, S.A.; Magnani, J.W.; Ellinor, P.T., et al. 50 year trends in atrial fibrillation prevalence, incidence, risk factors, and mortality in the Framingham Heart Study: a cohort study. Lancet 2015, 386, 154-162, doi:10.1016/S0140-6736(14)61774-8.

9. Kirchhof, P. The future of atrial fibrillation management: integrated care and stratified therapy. Lancet 2017, 390, 1873-1887, doi:10.1016/S0140-6736(17)31072-3.

10. Chiang, C.E.; Wang, K.L.; Lip, G.Y. Stroke prevention in atrial fibrillation: an Asian perspective. Thromb Haemost 2014, 111, 789-797, doi:10.1160/TH13-11-0948.

11. Heijman, J.; Voigt, N.; Nattel, S.; Dobrev, D. Cellular and molecular electrophysiology of atrial fibrillation initiation, maintenance, and progression. Circ Res 2014, 114, 1483-1499, doi:10.1161/CIRCRESAHA.114.302226.

12. Schmitt, N.; Grunnet, M.; Olesen, S.P. Cardiac potassium channel subtypes: new roles in repolarization and arrhythmia. Physiol Rev 2014, 94, 609-653, doi:10.1152/physrev.00022.2013.

13. Nattel, S.; Harada, M. Atrial remodeling and atrial fibrillation: recent advances and translational perspectives. J Am Coll Cardiol 2014, 63, 2335-2345, doi:10.1016/j.jacc.2014.02.555.

14. de Vos, C.B.; Pisters, R.; Nieuwlaat, R.; Prins, M.H.; Tieleman, R.G.; Coelen, R.J.; van den Heijkant, A.C.; Allessie, M.A.; Crijns, H.J. Progression from paroxysmal to persistent atrial fibrillation clinical correlates and prognosis. J Am Coll Cardiol 2010, 55, 725-731, doi:10.1016/j.jacc.2009.11.040.

15. Xiao, J.; Chen, Y.H. MicroRNAs: Novel Regulators of the Heart. J Thorac Dis 2010, 2, 43-47.

16. Wang, Z.; Lu, Y.; Yang, B. MicroRNAs and atrial fibrillation: new fundamentals. Cardiovasc Res 2011, 89, 710-721, doi:10.1093/cvr/cvq350.

17. Ambros, V. The functions of animal microRNAs. Nature 2004, 431, 350-355, doi:10.1038/nature02871.

18. Yang, D.; Wan, X.; Dennis, A.T.; Bektik, E.; Wang, Z.; Costa, M.G.S.; Fagnen, C.; Venien-Bryan, C.; Xu, X.; Gratz, D.H., et al. MicroRNA Biophysically Modulates Cardiac Action Potential via Directly Binding to Ion Channel. Circulation 2021, 10.1161/CIRCULATIONAHA.120.050098,

doi:10.1161/CIRCULATIONAHA.120.050098.

19. Han, Q.; Liu, D.; Convertino, M.; Wang, Z.; Jiang, C.; Kim, Y.H.; Luo, X.; Zhang, X.; Nackley, A.; Dokholyan, N.V., et al. miRNA-711 Binds and Activates TRPA1 Extracellularly to Evoke Acute and Chronic Pruritus. Neuron 2018, 99, 449-463 e446, doi:10.1016/j.neuron.2018.06.039.

20. Kwon, C.; Han, Z.; Olson, E.N.; Srivastava, D. MicroRNA1 influences cardiac differentiation in Drosophila and regulates Notch signaling. Proc Natl Acad Sci U S A 2005, 102, 18986-18991, doi:10.1073/pnas.0509535102.

21. Luo, X.; Pan, Z.; Shan, H.; Xiao, J.; Sun, X.; Wang, N.; Lin, H.; Xiao, L.; Maguy, A.; Qi, X.Y., et al. MicroRNA-26 governs profibrillatory inward-rectifier potassium current changes in atrial fibrillation. J Clin Invest 2013, 123, 1939-1951, doi:10.1172/JCI62185.

22. Li, H.; Gao, F.; Wang, X.; Wu, J.; Lu, K.; Liu, M.; Li, R.; Ding, L.; Wang, R. Circulating microRNA-378 levels serve as a novel biomarker for assessing the severity of coronary stenosis in patients with coronary artery disease. Biosci Rep 2019, 39, doi:10.1042/BSR20182016. 
23. Frangogiannis, N.G. Syndecan-1: a critical mediator in cardiac fibrosis. Hypertension 2010, 55, 233-235, doi:10.1161/HYPERTENSIONAHA.109.147256.

24. Lei, J.; Xue, S.; Wu, W.; Zhou, S.; Zhang, Y.; Yuan, G.; Wang, J. Sdc1 overexpression inhibits the p38 MAPK pathway and lessens fibrotic ventricular remodeling in MI rats. Inflammation 2013, 36, 603-615, doi:10.1007/s10753-012-9582-y.

25. Hu, Y.F.; Chen, Y.J.; Lin, Y.J.; Chen, S.A. Inflammation and the pathogenesis of atrial fibrillation. Nat Rev Cardiol 2015, 12, 230-243, doi:10.1038/nrcardio.2015.2.

26. Shannon, P.; Markiel, A.; Ozier, O.; Baliga, N.S.; Wang, J.T.; Ramage, D.; Amin, N.; Schwikowski, B.; Ideker, T. Cytoscape: a software environment for integrated models of biomolecular interaction networks. Genome Res 2003, 13, 2498-2504, doi:10.1101/gr.1239303.

27. Wang, T.; Wang, B. Identification of microRNA-mRNA interactions in atrial fibrillation using microarray expression profiles and bioinformatics analysis. Mol Med Rep 2016, 13, 4535-4540, doi:10.3892/mmr.2016.5106.

28. Chiang, D.Y.; Zhang, M.; Voigt, N.; Alsina, K.M.; Jakob, H.; Martin, J.F.; Dobrev, D.; Wehrens, X.H.T.; Li, N. Identification of microRNA-mRNA dysregulations in paroxysmal atrial fibrillation. Int J Cardiol 2015, 184, 190-197, doi:10.1016/j.ijcard.2015.01.075.

29. Jiang, S.; Guo, C.; Zhang, W.; Che, W.; Zhang, J.; Zhuang, S.; Wang, Y.; Zhang, Y.; Liu, B. The Integrative Regulatory Network of circRNA, microRNA, and mRNA in Atrial Fibrillation. Front Genet 2019, 10, 526, doi:10.3389/fgene.2019.00526.

30. Ruan, Z.B.; Wang, F.; Yu, Q.P.; Chen, G.C.; Zhu, L. Integrative analysis of the circRNA-miRNA regulatory network in atrial fibrillation. Sci Rep 2020, 10, 20451, doi:10.1038/s41598-020-77485-1.

31. Zhao, L.; Ma, Z.; Guo, Z.; Zheng, M.; Li, K.; Yang, X. Analysis of long non-coding RNA and mRNA profiles in epicardial adipose tissue of patients with atrial fibrillation. Biomed Pharmacother 2020, 121, 109634, doi:10.1016/j.biopha.2019.109634.

32. Ganesh, S.K.; Arnett, D.K.; Assimes, T.L.; Basson, C.T.; Chakravarti, A.; Ellinor, P.T.; Engler, M.B.; Goldmuntz, E.; Herrington, D.M.; Hershberger, R.E., et al. Genetics and genomics for the prevention and treatment of cardiovascular disease: update: a scientific statement from the American Heart Association. Circulation 2013, 128, 2813-2851, doi:10.1161/01.cir.0000437913.98912.1d.

33. Goldberg, I.J.; Eckel, R.H.; McPherson, R. Triglycerides and heart disease: still a hypothesis? Arterioscler Thromb Vasc Biol 2011, 31, 1716-1725, doi:10.1161/ATVBAHA.111.226100.

34. Corlin, L.; Liu, C.; Lin, H.; Leone, D.; Yang, Q.; Ngo, D.; Levy, D.; Cupples, L.A.; Gerszten, R.E.; Larson, M.G., et al. Proteomic Signatures of Lifestyle Risk Factors for Cardiovascular Disease: A Cross-Sectional Analysis of the Plasma Proteome in the Framingham Heart Study. J Am Heart Assoc 2021, 10, e018020, doi:10.1161/JAHA.120.018020.

35. Yoshida, M.; Takano, Y.; Sasaoka, T.; Izumi, T.; Kimura, A. E-selectin polymorphism associated with myocardial infarction causes enhanced leukocyte-endothelial interactions under flow conditions. Arterioscler Thromb Vasc Biol 2003, 23, 783-788, doi:10.1161/01.ATV.0000067427.40133.59.

36. Liao, B.; Chen, K.; Xiong, W.; Chen, R.; Mai, A.; Xu, Z.; Dong, S. Relationship of SELE A561C and G98T Variants With the Susceptibility to CAD. Medicine (Baltimore) 2016, 95, e1255, doi:10.1097/MD.0000000000001255.

37. Sandoval-Pinto, E.; Padilla-Gutierrez, J.R.; Valdes-Alvarado, E.; Garcia-Gonzalez, I.J.; Valdez-Haro, A.; Munoz-Valle, J.F.; Flores-Salinas, H.E.; Rivas, F.; Valle, Y. Assessment of the E-selectin rs5361 (561A>C) polymorphism and soluble protein concentration in acute coronary syndrome: association with circulating levels. Mediators Inflamm 2014, 2014, 158367, doi:10.1155/2014/158367. 
38. Li, Y.; Wei, Y.S.; Wang, M.; Zhang, P.A.; Jiang, X.J.; Huang, C.X. Association between the Ser128Arg variant of the E-selectin and risk of coronary artery disease in the central China. Int J Cardiol 2005, 103, 33-36, doi:10.1016/j.ijcard.2004.07.011.

39. Freestone, B.; Chong, A.Y.; Nuttall, S.; Blann, A.D.; Lip, G.Y. Soluble E-selectin, von Willebrand factor, soluble thrombomodulin, and total body nitrate/nitrite product as indices of endothelial damage/dysfunction in paroxysmal, persistent, and permanent atrial fibrillation. Chest 2007, 132, 1253-1258, doi:10.1378/chest.07-1185.

40. Freestone, B.; Chong, A.Y.; Nuttall, S.; Lip, G.Y. Impaired flow mediated dilatation as evidence of endothelial dysfunction in chronic atrial fibrillation: relationship to plasma von Willebrand factor and soluble E-selectin levels. Thromb Res 2008, 122, 85-90, doi:10.1016/j.thromres.2007.09.008.

41. Kwon, M.J.; Jang, B.; Yi, J.Y.; Han, I.O.; Oh, E.S. Syndecans play dual roles as cell adhesion receptors and docking receptors. FEBS Lett 2012, 586, 2207-2211, doi:10.1016/j.febslet.2012.05.037.

42. Couchman, J.R. Transmembrane signaling proteoglycans. Annu Rev Cell Dev Biol 2010, 26, 89-114, doi:10.1146/annurev-cellbio-100109-104126.

43. Akl, M.R.; Nagpal, P.; Ayoub, N.M.; Prabhu, S.A.; Gliksman, M.; Tai, B.; Hatipoglu, A.; Goy, A.; Suh, K.S. Molecular and clinical profiles of syndecan-1 in solid and hematological cancer for prognosis and precision medicine. Oncotarget 2015, 6, 28693-28715, doi:10.18632/oncotarget.4981.

44. Wang, X.; Lu, Y.; Xie, Y.; Shen, J.; Xiang, M. Emerging roles of proteoglycans in cardiac remodeling. Int J Cardiol 2019, 278, 192-198, doi:10.1016/j.ijcard.2018.11.125.

45. Shi, Q.; Jiang, J.; Luo, G. Syndecan-1 serves as the major receptor for attachment of hepatitis $C$ virus to the surfaces of hepatocytes. J Virol 2013, 87, 6866-6875, doi:10.1128/JVI.03475-12.

46. Teng, Y.H.; Aquino, R.S.; Park, P.W. Molecular functions of syndecan-1 in disease. Matrix Biol 2012, 31, 3-16, doi:10.1016/j.matbio.2011.10.001.

47. Palaiologou, M.; Delladetsima, I.; Tiniakos, D. CD138 (syndecan-1) expression in health and disease. Histol Histopathol 2014, 29, 177-189, doi:10.14670/HH-29.177.

48. Bertrand, J.; Bollmann, M. Soluble syndecans: biomarkers for diseases and therapeutic options. Br J Pharmacol 2019, 176, 67-81, doi:10.1111/bph.14397.

49. Chechi, K.; Voisine, P.; Mathieu, P.; Laplante, M.; Bonnet, S.; Picard, F.; Joubert, P.; Richard, D. Functional characterization of the Ucp1-associated oxidative phenotype of human epicardial adipose tissue. Sci Rep 2017, 7, 15566, doi:10.1038/s41598-017-15501-7.

50. Zhang, X.; Xie, H.; Chang, P.; Zhao, H.; Xia, Y.; Zhang, L.; Guo, X.; Huang, C.; Yan, F.; Hu, L., et al. Glycoprotein M6B Interacts with TbetaRI to Activate TGF-beta-Smad2/3 Signaling and Promote Smooth Muscle Cell Differentiation. Stem Cells 2019, 37, 190-201, doi:10.1002/stem.2938.

51. Park, J.H.; Pak, H.N.; Lee, S.; Park, H.K.; Seo, J.W.; Chang, B.C. The clinical significance of the atrial subendocardial smooth muscle layer and cardiac myofibroblasts in human atrial tissue with valvular atrial fibrillation. Cardiovasc Pathol 2013, 22, 58-64, doi:10.1016/j.carpath.2012.05.001.

52. Shin, O.H.; Han, W.; Wang, Y.; Sudhof, T.C. Evolutionarily conserved multiple C2 domain proteins with two transmembrane regions (MCTPs) and unusual Ca2+ binding properties. J Biol Chem 2005, 280, 1641-1651, doi:10.1074/jbc.M407305200.

53. Lalani, S.R.; Ware, S.M.; Wang, X.; Zapata, G.; Tian, Q.; Franco, L.M.; Jiang, Z.; Bucasas, K.; Scott, D.A.; Campeau, P.M., et al. MCTP2 is a dosage-sensitive gene required for cardiac outflow tract development. Hum Mol Genet 2013, 22, 4339-4348, doi:10.1093/hmg/ddt283.

54. Parker, L.E.; Landstrom, A.P. Genetic Etiology of Left-Sided Obstructive Heart Lesions: A Story in Development. J Am Heart Assoc 2021, 10, e019006, doi:10.1161/JAHA.120.019006. 
55. Verweij, N.; van de Vegte, Y.J.; van der Harst, P. Genetic study links components of the autonomous nervous system to heart-rate profile during exercise. Nat Commun 2018, 9, 898, doi:10.1038/s41467-018-03395-6.

56. van de Vegte, Y.J.; Tegegne, B.S.; Verweij, N.; Snieder, H.; van der Harst, P. Genetics and the heart rate response to exercise. Cell Mol Life Sci 2019, 76, 2391-2409, doi:10.1007/s00018-019-03079-4.

57. Leineweber, K.; Moosmang, S.; Paulson, D. Genetics of NO Deficiency. Am J Cardiol 2017, 120, S80-S88, doi:10.1016/j.amjcard.2017.06.013.

58. Schellings, M.W.; Vanhoutte, D.; van Almen, G.C.; Swinnen, M.; Leenders, J.J.; Kubben, N.; van Leeuwen, R.E.; Hofstra, L.; Heymans, S.; Pinto, Y.M. Syndecan-1 amplifies angiotensin II-induced cardiac fibrosis. Hypertension 2010, 55, 249-256, doi:10.1161/HYPERTENSIONAHA.109.137885.

59. Tromp, J.; van der Pol, A.; Klip, I.T.; de Boer, R.A.; Jaarsma, T.; van Gilst, W.H.; Voors, A.A.; van Veldhuisen, D.J.; van der Meer, P. Fibrosis marker syndecan-1 and outcome in patients with heart failure with reduced and preserved ejection fraction. Circ Heart Fail 2014, 7, 457-462, doi:10.1161/CIRCHEARTFAILURE.113.000846.

60. Morita, H.; Komuro, I. Heart Failure as an Aging-Related Phenotype. Int Heart J 2018, 59, 6-13, doi:10.1536/ihj.17-519.

61. Men, H.; Cai, H.; Cheng, Q.; Zhou, W.; Wang, X.; Huang, S.; Zheng, Y.; Cai, L. The regulatory roles of p53 in cardiovascular health and disease. Cell Mol Life Sci 2021, 78, 2001-2018, doi:10.1007/s00018-020-03694-6.

62. Kovacic, J.C.; Mercader, N.; Torres, M.; Boehm, M.; Fuster, V. Epithelial-to-mesenchymal and endothelial-to-mesenchymal transition: from cardiovascular development to disease. Circulation 2012, 125, 1795-1808, doi:10.1161/CIRCULATIONAHA.111.040352.

63. Krainock, M.; Toubat, O.; Danopoulos, S.; Beckham, A.; Warburton, D.; Kim, R. Epicardial Epithelial-to-Mesenchymal Transition in Heart Development and Disease. J Clin Med 2016, 5, doi:10.3390/jcm5020027.

64. Jesel, L.; Abbas, M.; Park, S.H.; Matsushita, K.; Kindo, M.; Hasan, H.; Auger, C.; Sato, C.; Ohlmann, P.; Mazzucotelli, J.P., et al. Atrial Fibrillation Progression Is Associated with Cell Senescence Burden as Determined by p53 and p16 Expression. J Clin Med 2019, 9, doi:10.3390/jcm9010036.

65. Xie, J.; Chen, Y.; Hu, C.; Pan, Q.; Wang, B.; Li, X.; Geng, J.; Xu, B. Premature senescence of cardiac fibroblasts and atrial fibrosis in patients with atrial fibrillation. Oncotarget 2017, 8, 57981-57990, doi:10.18632/oncotarget.19853.

66. Horn, M.A.; Trafford, A.W. Aging and the cardiac collagen matrix: Novel mediators of fibrotic remodelling. J Mol Cell Cardiol 2016, 93, 175-185, doi:10.1016/j.yjmcc.2015.11.005.

67. George, B.; Rivera Rolon, M.D.M.; Mohit, S.; Stevenson, H.L. Epithelial to mesenchymal transition in endomyocardial biopsies from orthotopic heart transplant recipients. BMJ Case Rep 2019, 12, doi:10.1136/bcr-2018-229175.

68. Guo, T.; Yu, W.; Lv, S.; Zhang, C.; Tian, Y. MiR-302a inhibits the tumorigenicity of ovarian cancer cells by suppression of SDC1. Int J Clin Exp Pathol 2015, 8, 4869-4880.

69. Zhang, J.; Cao, Z.; Ding, X.; Wei, X.; Zhang, X.; Hou, J.; Ouyang, J. The lncRNA XIST regulates the tumorigenicity of renal cell carcinoma cells via the miR-302c/SDC1 axis. Int J Clin Exp Pathol 2017, 10, 7481-7491.

70. Sattayaprasert, P.; Vasireddi, S.K.; Bektik, E.; Jeon, O.; Hajjiri, M.; Mackall, J.A.; Moravec, C.S.; Alsberg, E.; Fu, J.; Laurita, K.R. Human Cardiac Mesenchymal Stem Cells Remodel in Disease and Can Regulate Arrhythmia Substrates. Circ Arrhythm Electrophysiol 2020, 13, e008740, doi:10.1161/CIRCEP.120.008740.

71. Miftode, R.S.; Serban, I.L.; Timpau, A.S.; Miftode, I.L.; Ion, A.; Buburuz, A.M.; Costache, A.D.; Costache, II. Syndecan-1: A Review on Its Role in Heart Failure and Chronic Liver Disease Patients' Assessment. Cardiol Res Pract 2019, 2019, 4750580, doi:10.1155/2019/4750580. 
72. Zhang, H.; Tian, L.; Shen, M.; Tu, C.; Wu, H.; Gu, M.; Paik, D.T.; Wu, J.C. Generation of Quiescent Cardiac Fibroblasts From Human Induced Pluripotent Stem Cells for In Vitro Modeling of Cardiac Fibrosis. Circ Res 2019, 125, 552-566, doi:10.1161/CIRCRESAHA.119.315491.

73. Bektik, E.; Cowan, D.B.; Wang, D.Z. Long Non-Coding RNAs in Atrial Fibrillation: Pluripotent Stem Cell-Derived Cardiomyocytes as a Model System. Int J Mol Sci 2020, 21, doi:10.3390/ijms21155424.

74. Blazeski, A.; Zhu, R.; Hunter, D.W.; Weinberg, S.H.; Boheler, K.R.; Zambidis, E.T.; Tung, L.

Electrophysiological and contractile function of cardiomyocytes derived from human embryonic stem cells. Prog Biophys Mol Biol 2012, 110, 178-195, doi:10.1016/j.pbiomolbio.2012.07.012.

75. Ye, Q.; Liu, Q.; Ma, X.; Bai, S.; Chen, P.; Zhao, Y.; Bai, C.; Liu, Y.; Liu, K.; Xin, M., et al. MicroRNA-146b-5p promotes atrial fibrosis in atrial fibrillation by repressing TIMP4. J Cell Mol Med 2021, 10.1111/jcmm.16985, doi:10.1111/jcmm.16985.

76. Wang, J.; Wang, Y.; Han, J.; Li, Y.; Xie, C.; Xie, L.; Shi, J.; Zhang, J.; Yang, B.; Chen, D., et al. Integrated analysis of microRNA and mRNA expression profiles in the left atrium of patients with nonvalvular paroxysmal atrial fibrillation: Role of miR-146b-5p in atrial fibrosis. Heart Rhythm 2015, 12, 1018-1026, doi:10.1016/j.hrthm.2015.01.026.

77. Wang, J.; Ye, Q.; Bai, S.; Chen, P.; Zhao, Y.; Ma, X.; Bai, C.; Liu, Y.; Xin, M.; Zeng, C., et al. Inhibiting microRNA-155 attenuates atrial fibrillation by targeting CACNA1C. J Mol Cell Cardiol 2021, 155, 58-65, doi:10.1016/j.yjmcc.2021.02.008.

78. Cao, Y.; Cui, L. Identifying the key microRNAs implicated in atrial fibrillation. Anatol J Cardiol 2021, 25, 429-436, doi:10.14744/AnatolJCardiol.2020.41625.

79. Tran, K.V.; Majka, J.; Sanghai, S.; Sardana, M.; Lessard, D.; Milstone, Z.; Tanriverdi, K.; Freedman, J.E.; Fitzgibbons, T.P.; McManus, D. Micro-RNAs Are Related to Epicardial Adipose Tissue in Participants With Atrial Fibrillation: Data From the MiRhythm Study. Front Cardiovasc Med 2019, 6, 115, doi:10.3389/fcvm.2019.00115.

80. Freestone, B.; Lip, G.Y.; Chong, A.Y.; Nadar, S.; Lee, K.W.; Blann, A.D. Circulating endothelial cells in atrial fibrillation with and without acute cardiovascular disease. Thromb Haemost 2005, 94, 702-706, doi:10.1160/TH05-02-0093.

81. Huang, D.; Li, T.; Li, X.; Zhang, L.; Sun, L.; He, X.; Zhong, X.; Jia, D.; Song, L.; Semenza, G.L. HIF-1-mediated suppression of acyl-CoA dehydrogenases and fatty acid oxidation is critical for cancer progression. Cell reports 2014, 8, 1930-1942. 